Revue d'histoire de l'Amérique française

AG REVUE D.HISTOIRE DE L'AMÉRIQUE FRANÇAISE

\title{
La théorie du commerce principal chez MM. Creighton et Ouellet
}

\author{
Nigel Kent-Barber
}

Volume 22, numéro 3, décembre 1968

URI : https://id.erudit.org/iderudit/302798ar

DOI : https://doi.org/10.7202/302798ar

Aller au sommaire du numéro

Éditeur(s)

Institut d'histoire de l'Amérique française

\section{ISSN}

0035-2357 (imprimé)

1492-1383 (numérique)

Découvrir la revue

Citer cet article

Kent-Barber, N. (1968). La théorie du commerce principal chez MM. Creighton et Ouellet. Revue d'histoire de l'Amérique française, 22(3), 401-414.

https://doi.org/10.7202/302798ar d'utilisation que vous pouvez consulter en ligne.

https://apropos.erudit.org/fr/usagers/politique-dutilisation/ 


\section{LA THÉORIE DU COMMERCE PRINCIPAL CHEZ MM. CREIGHTON ET OUELLET}

Le livre Histoire économique et sociale du Québec 17601805 - Structures et conjonctures (Montréal, Fides, 1966) a reçu beaucoup de louanges. Nous sommes bien heureux d'y ajouter les nôtres, car l'histoire économique canadienne a un grand besoin d'études aussi fouillées et aussi scientifiques que celle du professeur Ouellet. N'empêche que son œuvre risque de secouer l'historiographie canadienne. Car, non seulement comprend-elle plus d'une critique sérieuse à l'endroit de ce grand historien contemporain qu'est M. D. G. Creighton, professeur émérite de l'Université de Toronto, mais aussi bien constitue-t-elle, par sa façon même d'aborder le sujet, une grave attaque contre la thèse la plus sacro-sainte de l'histoire économique canadienne, la fameuse staple trade theory ou théorie du commerce principal. ${ }^{1}$ Cette attaque, ainsi que ces critiques, méritent une analyse objective.

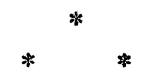

En bref, la théorie du commerce principal établit que l'histoire économique canadienne n'est pas en somme autre chose que l'histoire de l'exploitation de certaines matières brutes: la morue, les fourrures, le blé et le bois (certains historiens ajouteraient ici la potasse). Chacune de ces exploitations constituaient entièrement (les fourrures) ou en très grande partie

1 Le professeur Ouellet traduit "commerce d'étaple", qu'il semble avoir trouvé dans des documents du XIXe siècle. Nous n'avons aucune raison de suppaser qu'il s'agisse d'autre chose que d'un anglicisme, auquel la patine de l'âge ne saurait ajouter de la valeur. Le mot anglais staple n'a voulu dire, il y a cent cinquante ans, et ne veut dire aujourd'hui, que "plus important", "de base". Notre ami et collègue Jean-Guy Latulippe (du Département d'économique de l'Université de Sherbrooke) est d'accord avec nous pour rejeter cette fausse traduction et choisir plutôt celle du Dictionnaire canadien, conforme à celle des dictionnaires Harrap's et Larousse. 
(le bois) la production économique canadienne d'une époque. Jusqu'ici, tous les historiens, y compris le professeur Ouellet, sont d'accord. Mais lorsque la théorie du commerce principal confond en une seule et même chose l'histoire de ces exploitations et l'histoire économique du pays, M. Ouellet commence à n'être plus d'accord.

Le premier livre qui prend implicitement ce parti, c'est une petite monographie presque oubliée: Early Trading Companies of New France, paru en 1896, sous la signature d'un étudiant inconnu, E. P. Biggar. La thèse paraît tellement logique que le grand historien, feu Harold Innis, du "Department of Political Economy" de l'Université de Toronto, y appuie l'œuvre de sa vie. Tout le monde connaît son étude classique, The Fur Trade in Canada, achevée en 1930. Mais il convient de noter le sous-titre qu'a choisi Innis : An Introduction to Canadian Economic History (New Haven, Yale University Press). Cet ouvrage, encore irremplaçable, est suivi dix ans plus tard de The Cod Fisheries. The History of an International Economy (New Haven, Yale University Press, 1940).

Le livre pour lequel $M$. Ouellet réserve ses attaques les plus vives, The Empire of the St. Lawrence de D. G. Creighton (Toronto, MacMillan, 1956) est une réédition de The Commercial Empire of the St. Lawrence, publié en 1937 dans le cadre de la collection dite "The Relations of Canada and the United States", par The Ryerson Press pour le compte de Carnegie Endowment for International Peace. L'ouvrage se trouve donc - ne fût-ce que chronologiquement - dans la ligne des idées du professeur Innis; et la préface nous précise que l'auteur n'a pas changé d'idée depuis sa jeunesse jusqu'à la date de la réédition.

The book expounds and illustrates, through a narrative account, a certain thesis respecting Canada's historical development during a given period;... I should perhaps be inclined to modify slightly, here and there, some of the views expressed in this book; but the idea of the St. Lawrence as the inspiration and basis of a transcontinental, east-west system, 
both commercial and political in character, is still central in my interpretation of Canadian history. ${ }^{2}$ Much of my later work has been based upon the idea...

Nous avons dit "dans la ligne", c'est dire que pour nous, l'ouvrage de Creighton découle des idées d'Innis, qu'il accepte. Cette acceptation est la raison d'être de cet article. Si cette théorie n'était qu'une thèse parmi d'autres, nous nous serions contentés de faire remarquer comment s'y oppose l'ouvrage de M. Ouellet. Mais la thèse a fait son chemin au point qu'il n'est plus ni thèse ni école; c'est la base totalement acceptée de l'histoire économique canadienne. La constatation est hardie, dira-t-on. Mais notre avis est partagé par MM. Easterbrook et Aitken, compilateurs de Readings in Canadian Economic History (Ottawa, Carleton Library, 1966) qui ne font état que d'une seule attaque formelle, sous la forme d'un article de M. Fowkes, professeur à l'Université du Manitoba. Nous devons faire remarquer toutefois que M. Fowkes a publié par la suite une étude magistrale sur le commerce du blé, dans laquelle, effectivement, il ne s'écarte pas, lui non plus, de la ligne commune des historiens de l'économie canadienne. Pour le reste, nous ne pouvons que référer ceux qui s'intéressent à l'historiographie de la théorie du commerce principal aux remarques de MM. Easterbrook et Aitken. Ainsi, la contestation de M. Ouellet touche l'histoire économique canadienne tout entière, telle qu'elle est enseignée dans nos universités d'expression anglaise ou française.

A la page 150 de son Histoire économique et sociale du Québec 1760-1850, M. Ouellet entre dans le vif du sujet. Nous trouvons préférable de citer le passage au complet:

Les critiques que nous avons déjà formulées à l'endroit des interprétations du professeur Creighton s'appliquent aussi à sa vision de la décennie 1793-

${ }^{2}$ Même davantage, étant donnée l'extension qu'implique le changement de titre pour la réédition. 
1802. Dans son chapitre, The Rise of the New Staple Trades, il affirme: "The decade which followed the Constitutional Act of 1791 and the establishment of representative government in the Canada formed an important stage in the great transition through which the commercial state was passing. The decline of the fur-trading society in the region of the Great Lakes was now accompanied by the solid settlement of the fat, rich, forest-covered land. The rise of the new industries and the new staple trades was coincident with the beginnings of parliamentary governement. The uninterrupted onrush of change demanded constant effort and adjustment; it inspired the enterprising and perturbed the conservative; and it brought forward new and discredited old reputations." 3 Cette interprétation, extrêmement sensible aux modifications dans les structures, repose sur une acceptation trop facile de la "staple theory" et de la "frontier theory". Elle aboutit à une exagération du rôle joué par un Haut-Canada naissant et indique certaines lacunes dans la chronologie des phénomènes. En effet, c'est pendant la dernière décennie du siècle que le commerce des fourrures réalise ses meilleures performances et le déclin du trafic des pelleteries ne s'amorce vraiment qu'après 1800 . Même s'il n'emploie alors qu'une fraction secondaire de la population, il demeure la source principale de profits et l'instrument majeur par lequel s'affirme la bourgeoisie. On ne saurait minimiser son importance à l'âge d'or des marchands aventuriers sans rendre incompréhensibles certains cheminements en cours. Quant à l'économie du blé, elle n'est pas une création de ces dernières années. Nous en avons déjà indiqué la lente et constante association aux marchés extérieurs: pêcheries, Indes occidentales et GrandeGretagne. Si le professeur Creighton entend que l'accroissement du trafic des denrées agricoles a été tel qu'il a contribué à élever l'agriculture au rang de "staple production", nous sommes en grande partie d'accord. Les exportations de denrées agri-

3 D. G. Creighton, The Commercial Empire of the St. Lawrence 1760-1850 - The Relations of Canada and the United States (Toronto, 1937), 116. 
coles entre 1793 et 1802 tendent à égaler, sinon à dépasser, en valeur les envois de pelleteries. Mais pourquoi avoir sous-estimé la participation des basses terres du Saint-Laurent à ce trafic et avoir tant insisté sur la mise en place de l'agriculture du HautCanada ? Serait-ce que le Haut Saint-Laurent serait devenu le moteur essentiel de changements structuraux ? Le développement de la culture des céréales dans l'ouest débouchait, il est vrai, sur une amélioration des moyens de communications et menaçait l'économie du castor. En attendant, les producteurs locaux s'accommodèrent fort bien du marché créé par la traite des pelleteries et des conditions de navigation du fleuve. Ce ne sont pas les quelques milliers de quarts de farine envoyés chaque année au port de Québec par le Haut-Canada après 1793 qui pèsent lourd dans la balance. En 1802, la part du Haut-Canada, pourtant exceptionnellement élevée, dépasse à peine les $4 \%$ des envois globaux de blé et de farine. Comment peut-on croire, si ce n'est pas par l'application aveugle de la théorie des zones frontières, qu'une telle performance puisse justifier des conclusions aussi vastes ? Pendant la décennie 1793-1802, l'agriculture du Québec connaît enfin la prospérité. Avant de s'effondrer, elle enregistre ses performances les plus spectaculaires. A côté des pelleteries, elle est un facteur de progrès et de transformations et, si l'on veut, "un staple trade". En ce qui concerne le Haut-Canada, dont on ne doit pas sous-évaluer le devenir ni les issues nouvelles qu'il ouvrait, il en était encore à ses débuts. Mais, jusqu'en 1817 au moins, il fait plutôt figure de colonie du Bas-Canada. D'autre part, le commerce du bois, destiné à remplacer les fourrures comme pôle d'intégration économique et social, est un produit du Blocus continental. En 1793, personne n'aurait pu prédire, en raison de la modicité de cette entreprise, les élans spectaculaires de 1806.

Précisons: M. Ouellet reproche deux choses à M. Creighton. D'abord, son analyse; et ensuite, sa chronologie. Quant à son analyse, nous reprenons la phrase de M. Ouellet:

Cette interprétation, extrêmement sensible aux modifications dans les structures, repose sur une accep- 
tation trop facile de la "staple theory" et de la "frontier theory".

Que le professeur Creighton accepte la théorie du commerce principal, cela ne fait pas de doute. Mais que le chef de file de cette école d'interprétation de l'histoire économique canadienne soit d'accord avec la théorie américaine de l'expansionfrontière nous étonnerait fort! Les historiographes Easterbrook et Aitken (op. cit.) ont bien démontré que les historiens canadiens n'ont jamais voulu appliquer, dans le cadre canadien, ce système de développement continu par la mise en exploitation de nouvelles terres à la queue leu leu. D'ailleurs, comment le justifier ? La "frontière", dans le sens américain, n'a existé au Canada que pendant une trentaine d'années à l'époque de l'émigration massive vers le Haut-Canada; et encore pendant une vingtaine d'années - quatre-vingts ans plus tard! - vers les Prairies. Le système appliqué par les théoriciens des Etats-Unis dépend de l'exploitation continuelle d'une frontière sans cesse renouvelée, circonstance inconnue au Canada.

Nous croyons comprendre la raison de cette erreur par une phrase du professeur Creighton dans le chapitre en question:

New social groups appeared: old groups assumed a new prominence. The representative system opened a way of political action to the completely new class of agricultural frontiersmen in Upper Canada, as well as to the old and hitherto politically inactive group of French-Canadian farmers and professional men (p. 123).

Mais l'auteur - qui parle dans ce passage de l'opposition politique naissante aux milieux d'affaires dans les deux provinces, opposition aboutissant à la liaison étrange de Mackenzie et Papineau - insiste tout au long de ce chapitre sur les "staple trades", les commerces principaux. Cette "frontière" passagère du Haut-Canada ne sert dans son esprit qu'à élargir le champ d'action des hommes d'affaires montréalais et à étendre en conséquence leurs opérations commerciales. La théorie du commerce principal n'est ni perdue de vue ni remplacée par une théorie 
étrangère au contexte canadien. Tout simplement, elle change de cadre géographique.

It was the co-existence of the fur trade and the trade in the new staples which made the task of the merchants so novel and so difficult... Simcoe, who preferred agriculture to furs and frowned upon big monopolistic fur-trading companies, was delighted to find, when he visited Kingston late in 1794, that the merchants were already pretty deeply interested in other commercial projects. They were trying to exploit the new market as well as to preserve the old (pp. 119-120).

En deuxième lieu, le professeur Ouellet critique la chronologie de son collègue torontois.

Cette interprétation ... aboutit à une exagération du rôle joué par un Haut-Canada naissant et indique certaines lacunes dans la chronologie des phénomènes ( $\mathrm{p} .150)$.

Le reproche fait à $M$. Creighton, en somme, est celui de laisser de côté l'agriculture du Bas-Canada, d'exagérer le rôle tenu par celle du Haut-Canada et de faire de l'essor de cette dernière un événement de la fin du XVIIIe siècle et non des années 1820. Or, ces critiques sembleraient tout à fait justifiées et nous reprocherions nous-même à $M$. Creighton de ne pas porter plus d'attention à l'élan spectaculaire du commerce du blé québécois des années 1790-1800. Nous faisons en effet écho à M. Ouellet jusqu'à un certain point:

Mais pourquoi avoir sous-estimé la participation des basses terres du Saint-Laurent à ce trafic et avoir tant insisté sur la mise en place de l'agriculture du Haut-Canada?

Soit, l'on ne peut s'empêcher de s'interroger à cet égard. M. Ouellet va pourtant plus loin.

Serait-ce que le Haut Saint-Laurent serait devenu le moteur essentiel de changements structuraux (p. 151)?

Eh bien, non: M. Creighton ne l'a jamais prétendu. Il voit plutôt un changement structural dans le développement des 
nouveaux "staple trades" - le bois et le blé, dont avait besoin l'Angleterre assiégée - qui coïncide dans son esprit avec le déclin des pelleteries.

The decline of the fur-trading society in the region of the Great Lakes was now accompanied by the solid settlement of the fat, rich, forest-covered land (p. 116).

Précisons également qu'il y a tout un passage dans ce même chapitre de The Empire of the St. Lawrence dont le professeur Ouellet ne tient pas compte. Creighton ne possède pas les chiffres de Ouellet, c'est évident. Il sous-estime la production agricole québécoise, c'est exact. Mais il sait que l'exportation connaît un essor dans la dernière décennie du XVIIIe siècle. Et il sait aussi que la production haut-canadienne n'en forme qu'une petite fraction:

A good part of this wheat and flour was carried down the St. Lawrence, as the theorists of the new Canadian economy had always intended it should be, to swell the export from Montreal and Quebec... Upper Canada's portion of the Canadian export of wheat and flour was, of course, extremely small; but it grew rapidly during the first decade of the history of the province (pp. 120-121).

C'est dire que la critique de $M$. Ouellet repose, selon nous, sur un différend fondamental: différend d'optique chronologique et d'intérêts historiques. Prenons pour exemple les deux attaques contenues dans l'ouvrage de M. Ouellet aux pages 169 et 247. Dans la première, nous remarquons même une hésitation - le mot s'impose. Le passage commence ainsi :

La décennie 1803-1812 est une des plus importantes dans toute l'histoire du Québec (p. 169),

- c'est-à-dire qu'elle comprend la fin des commerces des fourrures et du blé dans la province. M. Ouellet cite une phrase de The Empire of the St. Lawrence:

The Canadian economic revolution, which had begun with the peace of 1783 and the coming of the Loyalists, reached and passed its half-mark in the first decade of the nineteenth century (p. 143). 
Or, quelle est la nuance ? Le professeur Ouellet fait cette analyse :

$\mathrm{Au}$ dire du professeur Creighton, cette période ne constituerait pourtant qu'une étape parmi plusieurs autres à l'intérieur d'un long processus de transformations, qui aurait touché peu à peu les structures économiques de la vallée ${ }^{4}$ du Saint-Laurent (p.169).

Il faudrait cependant relever que $M$. Creighton ne manque pas de signaler le caractère particulier de cette époque, qui n'est nullement pour lui non plus "une étape parmi plusieurs autres". Nous continuons la citation au point même où $\mathbf{M}$. Ouellet l'a laissée :

The Canadian economic revolution, which had begun with the peace of 1783 and the coming of the Loyalists, reached and passed its half-mark in the first decade of the nineteenth century. It was no longer possible to deny the changes which had taken place or to evade their implications. The new boundary, cutting across the old dominion of the St. Lawrence, was an established fact... The society based on settlement and the new staple trades was no longer a dream or a hypothesis. It was a fact. Something definitive had taken root... (p. 143).

Il serait ainsi plus exact de dire que, pour M. Creighton, cette décennie est la première d'un nouveau système économique. D'ailleurs, M. Ouellet paraît appuyer lui-même cette interprétation:

Sans nier les cheminements antérieurs, c'est-à-dire l'indispensable préparation, voulue ou non, qu'une économie se donne à chaque moment de son développement, il importe néanmoins de mettre en évidence le caractère révolutionnaire et mouvementé de cette période décisive (p. 169).

N'est-ce pas précisément ce que fait M. Creighton ? Il faut nuancer, a dit $M$. Ouellet, "cette interprétation qui, au total, insiste surtout sur les fluctuations semi-séculaires" (169). Soit.

4 Mais le professeur Creighton parle toujours de "l'empire du SaintLaurent", y compris la vallée, le bassin du fleuve et la province tout entière; en y ajoutant parfois le sud de l'actuel Ontario. Il pourrait cependant s'agir ici d'un simple lapsus de la part de M. Ouellet. 
Mais c'est néanmoins prêter au professeur Creighton une volonté d'interprétation systématique, détaillée - qui n'est point sa méthode de travail - à partir de statistiques dont il ne pouvait nullement disposer.

Nous faisons la même constatation à l'égard de la critique contenue dans les pp. 247-248 de l'étude de M. Ouellet. De nouveau, une critique plutôt imprécise: elle suggère une hésitation, tendant sous l'analyse à appuyer ce qu'elle critique. De nouveau, aussi, une différence surtout d'optique.

La liquidation de la compagnie $d u$ Nord-Ouest seraitelle le facteur le plus signifiant de la période 18151821 ? Le professeur Creighton, qui a consacré tout un chapitre à ce qu'il appelle "The End of the Fur Trade", l'a cru. Cette vue des choses nous semble discutable (p. 247).

Eh bien, il nous paraît qu'elle semblerait discutable pour le professeur Creighton, lui aussi ! Nous venons de relire le chapitre en question, et nous ne voyons nulle part une telle constatation. Le chapitre traite certainement de cette période, mais dans le cadre plus grand d'une section de son livre, ayant pour titre "Transition in the Region of the Lower Lakes", qui comprend, celle-ci, le déroulement des événements depuis 1783! Nous ne pouvons le redire trop souvent: l'ouvrage du professeur Creighton, thèse géographique divisée par grandes périodes thématiques, ne saurait se comparer à celui du professeur Ouellet, étude économique divisée par petites périodes statistiques. Nulle critique, basée sur cette dernière approche, ne peut donc être valide.

Continuons néanmoins la citation de M. Ouellet:

Le sort des pelleteries comme activité dominante était décidé depuis longtemps de sorte qu'on assiste au cours de ces années à l'aboutissement, incomplet il est vrai, de ce long processus de déclin (pp. 247-248).

Or, nous ne pouvons nous empêcher de trouver que ce commentaire ne fait que résumer les conclusions de M. Creighton 
dans ce même chapitre. Il commence, en effet, par une constatation brutale:

The War of 1812 was the final episode in the long struggle between settlement and the fur trade in the region of the Great Lakes (p. 175).

"Aboutissement" nous paraît, entre parenthèses, une excellente traduction de final episode.

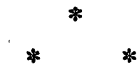

L'éclaircissement que nous avons tenté du bien et du malfondé de ces critiques n'est pourtant qu'un but secondaire de cet article. Il nous a paru néanmoins essentiel (ainsi qu'à notre ami et collègue $M$. Latulippe, à qui nous devons des conseils précieux) de "déblayer le terrain", pour ainsi dire, avant de considérer le sujet principal de notre étude. Car si M. Ouellet se livre à des attaques contre l'ouvrage de M. Creighton, il vise, nous paraît-il, tout le système basé sur la théorie du commerce principal, dont le professeur Creighton reste l'interprète le plus connu après le professeur Innis.

Porter atteinte à une interprétation universellement acceptée au Canada n'est manifestement pas le but de M. Ouellet; c'est néanmoins le résultat presque inévitable de ses énoncés. Dans le premier chapitre de l'Histoire économique et sociale du Québec 1760-1850, il affirme:

Notre objectif n'est pas d'écarter systématiquement tout ce qui s'est fait avant nous dans ce domaine encore si peu exploré de l'histoire économique et sociale. L'apport de l'historiographie du Canada anglais (sic) est trop considérable pour justifier une telle attitude. Les travaux de Innis, de Creighton, de Lower, de Brebner, de Cowan, de Tucker et de Easterbrook, pour ne nommer que les plus représentatifs, conservent toujours leur valeur. Il reste cependant qu'un renouvellement des perspectives s'impose et cela par le recours à de nouvelles méthodes - nouvelles pour nous - et à d'autres données que celles utilisées jusqu'à présent. Confronter ces travaux avec les séries chiffrées dont nous dispo- 
sions, essayer d'en approfondir les aspects positifs, les corriger au besoin, découvrir de nouvelles issues, toutes ces démarches nous ont permis d'en saisir à la fois l'intérêt et les lacunes. La précision relative à laquelle nous sommes parvenu a été un facteur primordial dans cet effort de rajeunissement, malheureusement bien modeste (p. 34).

Le lecteur aura noté que les historiens cités sont tous des disciples d'Innis dans ce domaine; tous des interprètes du commerce principal comme façon logique d'aborder l'histoire économique canadienne. La mise en cause devient ainsi, nous l'avons dit, inévitable. Les interprètes du soi-disant "Canada anglais" nous préférons le terme Canadiens anglophones pour éviter toute ambiguïté à l'égard de nos confrères dont la majorité ne sont pas de descendance anglaise - sont mis dans l'ombre par la statistique. Leur méthode, en l'absence de méthodes statistiques, se révélerait insuffisante. Si, par exemple, l'on analyse la critique que fait le professeur Ouellet ${ }^{5}$ de l'interprétation classique par Creighton de la décennie 1783-1792, on s'aperçoit que la différence en est une de méthodologie. Creighton ignore les cycles économiques - dont l'application possible à l'histoire économique canadienne ne nous a été révélée, d'ailleurs, que par l'analyse du professeur Ouellet de séries chiffrées découvertes en grande partie par lui seul. Et cela, trente années après la rédaction de The Commercial Empire of the St. Lawrence. M. Creighton ne saurait ainsi prendre le même parti.

M. Ouellet présente dans son ouvrage une analyse détaillée, serrée, suivant des cycles économiques dont la grande division se place en 1815. M. Creighton, par contre, nous propose une thèse - pas une analyse - sans détail ni analyse chronologique, dont les divisions, de nature bien moins scientifiques, se placent aux années 1783 et 1820. Monsieur Ouellet y voit, se trouve obligé d'y voir une insuffisance.

Tel est également le ton de la critique de la théorie elle-même. Une note sur le passage de $M$. Ouellet que nous venons de citer

${ }^{5}$ Histoire économique et sociale du Québec 1760-1850, 126. 
(sur la chronologie de M. Creighton) donne une définition des économistes canadiens Caves et Holton. ${ }^{6}$ Et M. Ouellet de préciser son attitude à l'égard de la théorie:

En dépit de ce qu'elle renferme d'éminemment juste, la "staple theory" ne nous semble pas devoir rendre compte de toute la réalité, même aux $18 \mathrm{e}$ et $19 \mathrm{e}$ siècles ... Nous croyons pouvoir accéder à une explication globale sans avoir à recourir d'une façon spécifique à ces deux théories (p. 150 n.).

Le professeur Ouellet prétend ne pas "avoir à recourir d'une façon spécifique" à la théorie du commerce principal. Est-ce exact? Il précise: "spécifique". Nous ne sommes pas spécialiste en sémantique, mais ce qualificatif ne nous semble avoir que fort peu de valeur dans un contexte de refus de la théorie. D'autre part, nous avons démontré que la théorie est généralement acceptée de nos jours. L'on chercherait en vain parmi l'œuvre d'Innis ou de Creighton un ouvrage sur la théorie du commerce principal, qu'ils ont pourtant acceptée et défendue par la méthode et par le contenu de leurs études. M. Ouellet s'en prend indirectement à une défense indirecte. L'histoire du commerce principal n'est-elle, en réalité, qu'une école parmi d'autres ... à défendre ou à attaquer parmi d'autres.

Nous posons done de nouveau la question. L'étude de M. Ouellet rejette-t-elle la méthodologie traditionnelle de la théorie du commerce principal ? Nous trouvons que non. Le professeur Ouellet, la trouvant insuffisante, la complète plutôt qu'il ne la rejette. Et la table des matières montre qu'il va en effet au-delà de l'histoire de ces exploitations de matière brute, rechercher une explication globale d'un ensemble économique global. Il ne saurait guère cependant nier avoir eu recours à la théorie.

Si nous examinons un peu la table des matières, nous constatons que la première des deux parties s'appelle "L'expan-

6 Qui, n'étant pas des historiens, la définissent en des termes plus contemporains que les nôtres. La définition que nous avons suggérée au début de cet article est d'ailleurs l'une des premières à paraître, pour les raisons avancées ici. 
sion par les fourrures et par le blé (1760-1815)" - titre de la plus pure tradition. Les chapitres portent des titres et des soustitres dont nous donnons un échantillon: "La rivalité MontréalAlbany dans le commerce des fourrures ..." "L'agriculture et le ravitaillement de l'armée..." "Le système commercial du SaintLaurent ..." "L'agriculture et le marché impérial ..." "La croissance du commerce du bois..." "L'agriculture et ses problèmes..." Il en est de même pour la deuxième partie: "La crise agricole et la baisse des prix..." "Relèvement de l'exploitation forestière et dépression dans la construction navale..." "Les prix agricoles ..." "Le régime britannique sauvé par le bois ..." "Les ressources de l'ouest et le déclin du vieux système colonial ..."

Les réalités historiques veulent en fait que l'on accepte le concept de base: l'économie canadienne a été tributaire de certaines grandes exploitations. Tel est le fait; nul historien économique ne saurait le nier. Mais nous avons fait remarquer que la théorie du commerce principal tend à mettre en équation, consciemment ou inconsciemment, l'exploitation - morue, fourrures, bois - et l'économie qui en dépend. Or, le professeur Ouellet refuse de s'engager dans cette voie. Telle est l'atteinte qu'il porte à la méthodologie traditionnelle canadienne.

Nigel KeNT-BARBeR

docteur de l'Université (Paris)

L'Index des dix dernières années de la Revue d'histoire de l'Amérique française, soit des vol. XI à XX est maintenant paru. On peut se le procurer en s'adressant à notre secrétariat. Le prix est de $\$ 6$. l'exemplaire. 